\title{
Joint optimization of phase diversity and adaptive optics: demonstration of potential
}

\author{
Visa Korkiakoski, ${ }^{1, \star}$ Christoph U. Keller, ${ }^{1}$ Niek Doelman, ${ }^{2}$ \\ Rufus Fraanje, ${ }^{3}$ and Michel Verhaegen ${ }^{3}$ \\ 'Utrecht University, Princetonplein 5, 3584CC Utrecht, The Netherlands \\ ${ }^{2}$ TNO Science and Industry, Stieltjesweg 1, 2628CK Delft, The Netherlands \\ ${ }^{3}$ Delft Center for Systems and Control, Mekelweg 2, 2628CD Delft, The Netherlands \\ ${ }^{*}$ Corresponding author: v.a.korkiakoski@uu.nl
}

Received 11 July 2011; revised 16 September 2011; accepted 22 September 2011; posted 27 September 2011 (Doc. ID 150787); published 23 December 2011

\begin{abstract}
We study different possibilities to use adaptive optics (AO) and phase diversity (PD) together in a jointly optimized system. The potential of the joint system is demonstrated through numerical simulations. We find that the most significant benefits are obtained from the improved deconvolution of AO-corrected wavefronts and the additional wavefront sensor (WFS) information that reduces the computational demands of PD algorithms. When applied together, it is seen that the image error can be reduced by $20 \%$ compared to traditional PD, working with one focused and one defocused camera image, and the computational load is reduced by a factor of 20 compared to a more reliable PD algorithm requiring more camera images. In addition, we find that the system performance can be optimized by adjusting the magnitude of the applied diversity wavefronts. (C) 2011 Optical Society of America
\end{abstract}

OCIS codes: $\quad 100.3010,100.3190,100.1830,010.1080$.

\section{Introduction}

When distant objects are imaged through turbulent air, such as in ground-based astronomy or with longrange surveillance cameras, the image quality suffers from distortions of the wavefronts after having passed through the turbulent atmosphere. Wellestablished techniques exist to compensate these distortions: a set of short-exposure images can be postprocessed to achieve higher image quality, or the distortions can be corrected in real-time using adaptive optics (AO) [1].

These two methods to improve the image quality rely on different principles: one tries to estimate the point-spread functions (PSF) of the distorted images to reconstruct the original object through deconvolution, and the other uses techniques to obtain a better PSF by correcting the distorted wavefront

$1559-128 \mathrm{X} / 12 / 010102-12 \$ 15.00 / 0$

(C) 2012 Optical Society of America
(WF) in real-time. Both the PSF estimation techniques and $\mathrm{AO}$ have been developed independently over several decades with good results.

Both techniques can be applied in a wide range of conditions: from pointlike dim sources of nighttime astronomy to bright, extended objects confronted when imaging the solar surface. However, particularly in the latter case, the current state-of-the-art systems are still having difficulties reaching the diffraction limited image quality in difficult circumstances, at visible wavelengths and when imaging wide fields of view.

Postprocessing techniques matured before technology had advanced enough to realize the more demanding real-time AO. One of the most powerful techniques, applicable also for infinitely extended sources, is phase diversity (PD). It makes it possible to invert the imaging problem-solve for the distorting WFs and PSFs from intensity images-by introducing known phase differences between simultaneous images. The technique was proposed in the 
1980s [2], originally to be used with two images, one focused and another having a known defocus. However, in many cases, two images are not sufficient, since the observed images do not necessarily contain information to reconstruct the original object for all spatial frequencies.

Therefore, the PD methods have been developed further by several authors to include information also from multiple frames [3-5] and multiple objects (in practice the solar surface at different wavelengths) $[6,7]$. In addition, recent work has been done by others, for instance, to illustrate how the WF $a$ priori information can be taken into account to increase the robustness of the reconstruction [8], or to demonstrate that the PD methods can be improved also by modeling the scintillation [9]. However, PD techniques remain computationally very demanding, and thus their use is mostly restricted to postprocessing.

Although AO has been successfully used in solar astronomy [10], difficult issues are faced to further improve AO performance. For instance, to measure the WF accurately, it is necessary to increase the sampling, most often by decreasing the ShackHartman wavefront sensor (SH-WFS) subaperture size. This, however, increases the blurring of the SH-WFS spots, and thus the correlation tracking becomes more difficult since the solar granules cannot be resolved. Therefore, solar AO has a fundamental limit in improving the image contrast. Besides, a simple, single conjugate AO system is also limited by the size of the anisoplanatic patch, which makes it difficult to correct a large field of view without complicated systems like multiconjugate adaptive optics (MCAO) [11].

The combination of $\mathrm{AO}$ and postprocessing techniques like PD, however, has not been well studied before. A simple AO system typically works in a closed-loop operation, where the incoming WF, after being reflected by a deformable mirror (DM), is divided between wavefront sensor (WFS) and imaging camera. The WF is reconstructed from the WFS measurements, and iterative DM corrections are applied to minimize the residual WF variations. Attempts have been made to deconvolve the long-exposure scientific images with PSFs reconstructed from the control data [12], and image restoration techniques have been applied independently to AO-corrected images [13-16].

However, several possibilities exist to enhance the capabilities of a combined AO and PD system. For instance, the DM itself can be used to introduce the diversity shape, as demonstrated in calibrating the noncommon path errors in AO systems [17] and also suggested by $[18,19]$. This makes it possible to dynamically introduce adjusted diversity shapes, based on current seeing conditions, and these are not restricted to defocus only. It is also not necessary to correct all the turbulence with the DM-the AO control algorithm should operate such that the outcome of the PD will provide an optimal result. In addition, the WFS information can be fed into the $\mathrm{PD}$ algorithms to provide useful knowledge of the WF shapes and reduce the computational demands.

This work is a first step to investigate issues when designing jointly optimized AO systems using PD. We investigate the potential to improve the image quality toward the diffraction limit, in particular in cases where infinitely extended, bright sources are imaged through a turbulent medium.

Section 2 presents the details of our PD algorithm, incorporating most of the advances of recent years. Then, in Section 3, we demonstrate through numerical simulations the potential of the joint optimization. Finally, Section $\underline{4}$ discusses our results and presents ideas for future efforts.

\section{Applied PD Method}

Several implementations of PD have been published in the past $[\underline{3}, \underline{4}, \underline{7}-\underline{9}, \underline{20}]$, and some of them are freely available, for instance [7].

In our work, we have followed the approach of Löfdahl and Scharmer [4] and incorporated some of the recent $\mathrm{PD}$ improvements. The main differences and additions are the following:

- We include multiple images and phase diversities in the same manner as in [3].

- We include a priori information of the WFs as done by [8].

- Instead of simultaneous imaging channels, images are collected at subsequent time steps, and thus it can be necessary to estimate the WFs independently.

Our implementation of PD permits us to explicitly and conveniently include most of the functionality presented in the multiobject multiframe blind deconvolution (MOMFBD) code [7]. In particular, we can easily include additional $\overline{\mathrm{W} F}$ information to aid the algorithm. The details of the algorithm are described in the following.

\section{A. Notations and Assumptions}

We assume that instead of multiple imaging channels, the intensity images $\left(d_{i}\right)$ are taken with a single camera at different time steps $\left(t_{i}\right)$, and the diversity is produced by a DM. We collect $N$ short-exposure images, and each is assumed to represent the same object $(f)$ with a unique PSF. The resulting images are then smeared by unknown WFs $\left(\phi_{i}\right)$ and known diversity components $\left(\psi_{i}\right)$. The WFs are partially known from the real-time WFS information obtained from an $\mathrm{AO}$ system.

The data collection scheme is illustrated in Fig. 1 , and the notations are summarized in Table 1.

As shown in Fig. 1, the most general approach for the PD problem is to assume $N$ arbitrary WFs for $N$ intensity images. Unfortunately, this would result in a problem that cannot be fully inverted. However, if the frame-rate of the imaging camera is sufficiently fast, it is an acceptable approximation that a few sequential WFs are assumed to be identical 


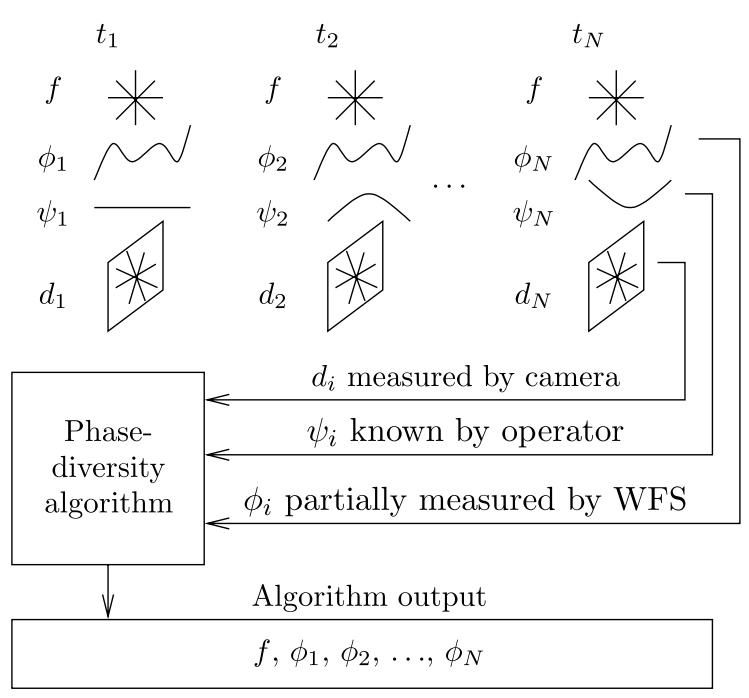

Fig. 1. Data collection scheme in our PD algorithm.

$\left(\phi_{1}=\phi_{2}=\cdots=\phi_{N}\right)$-an approach we mostly use in this work. When collecting multiple images, it is also possible to decide which particular WFs should be estimated independently.

At this moment, our algorithm does not define any fixed strategy to determine how to select the number of used images or the number of independently estimated WFs $(K)$. Instead, we will use different approaches to test which parameters are most promising. For instance, as we have done in this work in one scenario, we collect four images $(N=4)$ and estimate two WFs independently $(K=2)$ such that $\phi_{1}=\phi_{2}$ and $\phi_{3}=\phi_{4}$.

B. Numerical Approach to Invert the Imaging Problem

We follow the conventional approach and solve the estimation problem by minimizing the error metric similarly to that proposed by [2] ,

$$
L=\sum_{k=1}^{N} \sum_{u, v} \gamma_{k}\left|D_{k}-\hat{F} \hat{T}_{k}\right|^{2}+Z
$$

Table 1. Used Notations

\begin{tabular}{ll}
\hline$N$ & number of observed images \\
$K$ & number of considered WF estimations \\
$M$ & number of modes used by PD algorithm \\
$M^{\prime}$ & number of modes estimated from WFS signal \\
$M_{k}(x, y)$ & mode shape \\
$d_{k}, D_{k}$ & observed image, same in Fourier space \\
$t_{k}$ & PSF \\
$T_{k}$ & optical transfer function \\
$f, F$ & object, same in Fourier space \\
$\sigma_{k}$ & rms of noise in observed image \\
$\gamma$ & $1 / \sigma^{2}$ \\
$c_{i l}$ & modal coefficient (ith mode, $l$ th WF) \\
$r_{i}$ & $a$ priori known modal rms in WF \\
$P(x, y)$ & pupil shape \\
$\phi_{k}(x, y)$ & unknown WF distortion \\
$\psi_{k}(x, g)$ & known WF diversity \\
$\epsilon_{r}, \epsilon_{s}$ & relative reconstruction/image error \\
\hline
\end{tabular}

where $(u, v)$ are the Fourier space coordinates, $\gamma_{k}=\frac{1}{\sigma_{k}^{2}}$ ( $\sigma_{k}$ being the rms of noise in the $k$ th observed image), $F$ is the Fourier transform of the object (denoting an estimate), $T_{k}$ is the transfer function of the PSF in the $k$ th image, and $Z$ is the adjustment to take into account a priori knowledge of the WF.

The first term in Eq. (1) describes the sum of squared differences between the observed images and their estimates, derived from object and PSFs, exactly as done in [3]. The second term takes into account the $a$ priori information in the same manner as done in []ㅡ,

$$
Z=\sum_{l=1}^{K} \sum_{i=1}^{M} r_{i}^{2} c_{i l}^{2}
$$

where $r_{i}^{2}$ is the a priori known variance of the $i$ th mode in the WF, $M$ is the number of modes used by the PD algorithm, and $c_{i l}$ is the estimate of the $i$ th modal component in the $l$ th estimated WF. The covariances of WFs are neglected, which makes sense if the used modal base is close enough to KarhunenLoeve (KL) modes known to be statistically independent [21].

The transfer functions $T_{k}$ are defined as Fourier transforms of the PSFs, which we model as a function of the WF distortions as

$$
t_{k}(x, y)=\left|\mathcal{F}\left\{P(x, y) \exp \left[i\left(\phi_{l}(x, y)+\psi_{k}(x, y)\right)\right]\right\}\right|^{2},
$$

where $P(x, y)$ is a constant pupil function, $\psi_{k}(x, y)$ is the $k$ th known introduced diversity shape, $\phi_{l}(x, y)$ is the corresponding unknown WF aberration, and $\mathcal{F}\{\cdot\}$ denotes the Fourier transform.

The estimated WF, $\phi_{l}(x, y)$, is assumed to be known partially via other means, in practice by reconstruction from the WFS signal. Thus, using a modal composition, the WF can be written as

$$
\phi_{l}(x, y)=\sum_{i=1}^{M} M_{i}(x, y) c_{i l}+\sum_{i^{\prime}=1}^{M^{\prime}} M_{i^{\prime}}^{\prime}(x, y) c_{i^{\prime} l}^{\prime},
$$

where $M$ and $M^{\prime}$ are the number of used modes (estimated by PD and known from WFS signal), and $M_{i}(x, y)$ and $M_{i}^{\prime}(x, y)$ are the corresponding modal shapes. The first term describes the component estimated by PD, and the second component is known from the WFS information. In our work, we use a set of KL modes such that $M_{i}^{\prime}(x, y)$ are typically 0 20 first modes containing mainly low spatial frequencies, and $M_{i}(x, y)$ are the subsequent higher-order modes.

In the following, we assume that the coefficients estimated from the WFS signal are treated as constants. This is reasonable since the WF reconstruction using conventional $\mathrm{AO}$ techniques is computationally very fast compared to the PD. Therefore, the PD algorithm needs to estimate only $M$ coefficients. 
As shown by $[2,3]$, the object expressed as a function of observations and transfer functions,

$$
F_{M}=Q^{2} \sum_{k=1}^{N} \gamma_{k} D_{k} \hat{T}_{k}^{*}
$$

where

$$
Q=\left(\sum_{k=1}^{N} \gamma_{k}\left|T_{k}\right|^{2}\right)^{-1 / 2},
$$

can be substituted into Eq. (1) to obtain an the error metric with a radically lower number of unknown parameters,

$$
\begin{aligned}
L_{M}= & \sum_{j=1}^{N-1} \sum_{k=j+1}^{N} \sum_{u, v} Q^{2} \gamma_{j} \gamma_{k}\left|D_{j} \hat{T}_{k}-D_{k} \hat{T}_{j}\right|^{2} \\
& +\sum_{l=1}^{K} \sum_{i=1}^{M} r_{i}^{2} c_{i l}^{2} .
\end{aligned}
$$

This error metric can be considered as a sum of pairwise formed subtractions,

$$
L_{M}=\sum_{j=1}^{N-1} \sum_{k=j+1}^{N} \sum_{u, v}\left|E_{j k}\right|^{2}+\sum_{l=1}^{K} \sum_{i=1}^{M} r_{i}^{2} c_{i l}^{2},
$$

where

$$
E_{j k}=Q\left(\gamma_{j} \gamma_{k}\right)^{1 / 2}\left(D_{j} \hat{T}_{k}-D_{k} \hat{T}_{j}\right)
$$

In the same manner as done in [4], we can compute the partial derivatives of $E_{j k}$ with respect to the unknown parameters,

$$
\begin{aligned}
\frac{\partial E_{j k}}{\partial c_{i l}}= & \left(\gamma_{j} \gamma_{k}\right)^{1 / 2}\left[D_{j}\left(Q \frac{\partial \hat{T}_{k}}{\partial c_{i l}}+\frac{\partial Q}{\partial c_{i l}} \hat{T}_{k}\right)\right. \\
& \left.-D_{k}\left(Q \frac{\partial \hat{T}_{j}}{\partial c_{i l}}+\frac{\partial Q}{\partial c_{i l}} \hat{T}_{j}\right)\right],
\end{aligned}
$$

where

$$
\frac{\partial Q}{\partial c_{i l}}=-Q^{3} \operatorname{Re}\left(\sum_{k=1}^{N} \gamma_{k} \hat{T}_{k}^{*} \frac{\partial \hat{T}_{k}}{\partial c_{i l}}\right)
$$

and

$$
\frac{\partial \hat{T}_{k}}{\partial c_{i l}}=0 \quad \text { if } k \neq l .
$$

The parameters minimizing the error metric can then be found by iterative updating. We use, due to the simplicity of the implementation, the method suggested in [4], where the updates $\Delta c_{i l}$ are designed to minimize the formula

$$
\begin{aligned}
& \sum_{j=1}^{N-1} \sum_{k=j+1}^{N} \sum_{u, v}\left|E_{j k}+\sum_{l=1}^{K} \sum_{i=1}^{M} \frac{\partial E_{j k}}{\partial c_{i l}} \Delta c_{i l}\right|^{2} \\
& \quad+\sum_{l=1}^{K} \sum_{i=1}^{M} r_{i}^{2}\left(c_{i l}+\Delta c_{i l}\right)^{2} .
\end{aligned}
$$

This yields the matrix equation,

$$
A \Delta c+b=0,
$$

where the elements of $A$ and $b$ can be computed as

$$
A\left(m, m^{\prime}\right)=\sum_{j=1}^{N-1} \sum_{k=j+1}^{N}\left(\frac{\partial E_{j k}}{\partial c_{i l}} \mid \frac{\partial E_{j k}}{\partial c_{i^{\prime} l^{\prime}}}\right)+\delta\left(m, m^{\prime}\right) r_{i}^{2}
$$

and

$$
b(m)=\sum_{j=1}^{N-1} \sum_{k=j+1}^{N}\left(\frac{\partial E_{j k}}{\partial c_{i l}} \mid E_{j k}\right)+r_{i}^{2} c_{i l},
$$

where $(\cdot \mid \cdot)$ denotes an inner product, $\delta\left(m, m^{\prime}\right)$ is Kronecker's delta, $m=i+M(l-1)$, and $m^{\prime}=$ $i^{\prime}+M\left(l^{\prime}-1\right)$. Indices $i, i^{\prime}$ enumerate the estimated modes $\left(1 \leq i, i^{\prime} \leq M\right)$, and indices $l, l^{\prime}$ enumerate the independently estimated WFs $\left(1 \leq l, l^{\prime} \leq K\right)$. Thus matrix $A$ has a size of $K M \times K M$.

When $N=2, K=1, M^{\prime}=0$, and $r_{i}=0$ (two images, one unknown WF, no WFS or a priori information available), the algorithm is identical to what is described by Löfdahl and Scharmer in [4].

To prevent abrupt changes of the coefficients, we always did the inversion of $A$ via singular value decomposition and filtered out eigenvalues below $10^{-2}$. The simulations showed that this method worked well in all practical cases we encountered.

Our approaches to cope with the fast Fourier transform (FFT) border effects and image noise also follow the principles presented in [4]. The subimages are low-pass filtered to remove sharp edges, and the inner products in $A$ and $b$ are computed in image space: the values of $\frac{\partial E_{j k}}{\partial c_{i l}}$ are computed as shown by Eq. (10), and then the image space errors are obtained by FFTs. When performing the deconvolution in Eq. (5), a modified Wiener filter was applied with the same parameters as used in [4].

\section{Simulation Results}

In this section, we show our simulation results demonstrating the capabilities of the PD combined with AO. We will select a few relevant scenarios, and then determine the system performance by comparing the reconstruction performance with the original object.

A typical AO setup and our flexible $\mathrm{PD}$ algorithm offer many possibilities to use PD. In this work, we will use two options:

- 2-image PD: conventional PD with two images, one focused and another defocused 
- 4-image PD: phase diversity with four images, the first focused and the other having diversity shapes following the low-order KL modes, similar to the static Zernike polynomials such as astigmatism and coma [22]

The magnitudes of the applied diversities will be optimized for the given WF statistics, as discussed in Subsection 3.A. We tested other options (different shapes and number of used images) as well, but no particular method proved more promising.

Our approach also requires that the number of modes estimated from the WFS information is adjusted manually before running the PD algorithm. We will demonstrate the system performance with two options:

- traditional PD, where only tip/tilt information is obtained via other means, as it cannot be detected by the error metric in Eq. (1) $\left(M^{\prime}=0\right)$

- a case representative of a low-order SH-WFSthe first 20 lowest-order modal coefficients are obtained from the WFS signal $\left(M^{\prime}=20\right)$

The quality of the system performance will be assessed by comparing the PD reconstruction with the original object. We use a relative image error,

$$
\epsilon=\left[\frac{\sum\left(I_{r}-I\right)^{2}}{\sum I^{2}}\right]^{1 / 2},
$$

where the summation is done over the pixels, $I_{r}$ is the reconstructed (or seeing-limited) image, and $I$ is the original object.

In this work, we use a low-contrast object (rms contrast [23] is 0.19), shown later in Subsection 3.C. It is a synthetic image of the solar surface, derived from the simulation described in [24]. The observed images (dimension of $512 \times 512$ ) are simulated by convolving the object with a PSF that depends on the WF distortions. Then, we add random noise having an rms of $0.33 \%$ of the mean image intensity of the simulated images.

We have assumed that the DM is able to produce known diversity shapes with an error that is negligible compared to other sources.

The $a$ priori known modal rms $\left(r_{i}\right)$, as in Eq. (2), is used both for open and closed-loop data, and we obtain the rms values through a numerical approach. At first, the power spectral density of the residual WF is estimated using a method described in [25]. Then, we project several random realizations of corresponding WFs on the used modes and estimate their modal variance.

The final reconstruction is then compiled by mosaicing the subimages, which, due to the guard-band, has a dimension of $384 \times 384$ pixels. In this work, where all the subimages share the same aberrations, the mosaicing is not vital for dealing with anisoplanatism. However, since the subimages have different scenes, the mosaicing works as an additional robustness check of the PD algorithms. When evaluating
Table 2. AO Simulation Parameters

\begin{tabular}{ll} 
Telescope diameter & $1 \mathrm{~m}$ \\
Central obstruction & no \\
$\lambda_{\text {WFS }}$ & $800 \mathrm{~nm}$ \\
$\lambda_{\text {IMG }}$ & $550 \mathrm{~nm}$ \\
PSF resolution & $\lambda / D \sim 2$ pixels \\
WFS type & SH-WFS \\
Subapertures & $8 \times 8,52$ active \\
Frame-rate & $400 \mathrm{~Hz}$ \\
Servo lag & 2 frames \\
Controller & simple integrator \\
DM & 35 Zernike modes corrected \\
Turbulence model & von Karman \\
$r_{0}$ at $0.5 \mu \mathrm{m}$ & $0.08 \mathrm{~m}$ \\
Outer scale $L_{0}$ & $20 \mathrm{~m}$ \\
Number of layers & 2 \\
Wind speeds & $5 \mathrm{~m} / \mathrm{s}$ \\
Evolution time $\tau_{0}$ & $3.1 \mathrm{~ms}$ \\
Simulated time steps & 256 \\
\hline
\end{tabular}

the reconstruction quality, we use only the subimages to obtain more samples for the relevant statistics.

The simulation parameters are shown in Table 2. We consider a 1 meter telescope with a simple SH-WFS and a DM capable of correcting the 35 first Zernike modes.

Subsections 3.A and 3.B study the theoretical upper limit of the performance. We create random WF realizations, both with atmospheric turbulence and AO-corrected characteristics. As PD cannot detect tip/tilt, it is removed before the PD algorithm. We will assume that there is no time-lag between different diversity realizations (both in the simulated data and PD computations) and no error is made when WFs are estimated from WFS information.

Subsection 3.C demonstrates the performance with more realistic AO data. We will use images typical for a simple AO system, and we see how good performance can be reached. We include both timelag and typical WFS errors.

\section{A. Optimal Diversity Magnitude and Shape}

At first, we studied what kind of diversity shape would be optimal when the unknown WF is a typical Kolmogorov-style atmospheric distortion. As the DM is able to provide arbitrary diversity shapes, it could theoretically be possible to find shapes that outperform the conventional defocus diversity. However, we concluded that it would be extremely challenging to find such shapes. A similar problem has been addressed earlier by [26], and it was concluded that no particular diversity shape is a priori better than the others.

Regarding the diversity magnitude, a few studies can be found in the literature dealing with, for instance, an optimal defocus distance in the conventional PD [27-29]. However, less attention has been paid on how the multi-image PD will change the requirements for optimality and robustness. We made a few simulations and concluded that the PD 
algorithm is very robust in a wide range of applied diversity magnitudes.

Figure 2 shows the results, where the PD performance in atmospheric turbulence is plotted as a function of applied diversity rms. We have tested both the 2-image and 4-image $\mathrm{PD}$, and used three seeing values $\left(r_{0}\right.$ is $0.05,0.08$, and $0.15 \mathrm{~m}$ at $500 \mathrm{~nm}$ ). In those seeing conditions, the rms values of the turbulence induced WFs were $7 \pm 3,5 \pm 2$, and $3 \pm 1$ radians, respectively.

The smallest image error is always about $2 / 3$ of the seeing-limited case, independent of the seeing. The ratio depends on the proportion of the high and low spatial frequencies in the WF. The more nonestimable high spatial frequency componentsacting as a noise in the reconstruction algorithmthe worse the PD performance. However, it is possible to improve the optimal reconstruction quality at the cost of increased computational demand, as will be shown in Subsection 3.B.
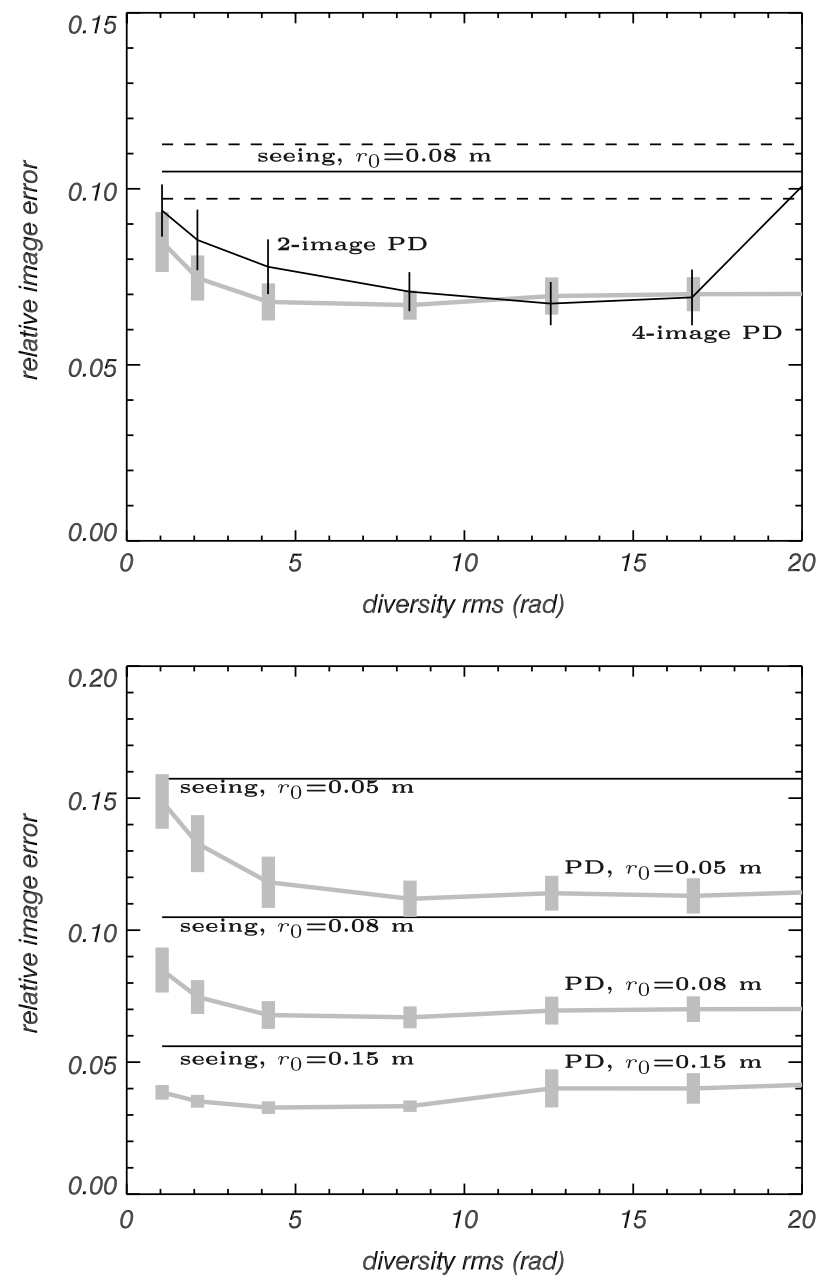

Fig. 2. Relative image reconstruction error as a function of applied diversity rms. $M=36, M^{\prime}=0$. Horizontal lines show the original image error. Error bars show the range of image error in terms of rms computed from different realizations (9 WFs times 9 images). Thin lines: 2-image PD used. Thick gray lines: 4-image PD used. Upper plot: $r_{0}$ is $0.08 \mathrm{~m}$ (the range of original image error shown by dashed lines). Lower plot: $r_{0}$ is $0.05,0.08$, and $0.15 \mathrm{~m}$.
The upper plot in Fig. 2 shows that as long as the applied diversity $\mathrm{rms}$ is approximately between 5 $17 \mathrm{rad}$, an optimal image reconstruction performance is obtained. The reconstruction is more robust with the 4-image $\mathrm{PD}$, in particular in the sense that the applied diversity magnitude is not very important. We did similar studies also with other KL modes as diversity, but the results were quantitatively not different than what is shown here. The lower plot in Fig. 2 shows that similar, very robust reconstructions at a wide range of diversity magnitudes can easily be obtained for $r_{0}$ (at $500 \mathrm{~nm}$ ) in the range of $0.05-0.15 \mathrm{~m}$.

It can be concluded that the applied diversity rms should be approximately as big as the unknown WF rms. However, as long as the diversity falls into a wide, slightly seeing-dependent interval, the reconstructions will be good. In extremely good seeing, the optimal results are obtained with magnitudes of 3-8 rad, while in bad seeing, higher magnitudes (8-15 rad) are needed.

We also tested what happens if similar simulations are made with more estimated modes ( $M$ up to 126). It was observed that the diversity magnitude ranges, where optimal reconstruction is obtained, will get narrower as the number of estimated modes is increased-in particular, at large diversity magnitudes, the reconstructions will fail more often. Thus, it might be necessary to optimize the applied diversity magnitude to guarantee optimal reconstruction performance.

These conclusions are in good agreement with the literature.

\section{B. Reconstruction Quality Versus Computational Needs}

The PD algorithm has two main parameters that can be adjusted to improve the reconstruction quality at the expense of higher computational demands: the number of estimated modes $(M)$ and the number of used images $(N)$. Unfortunately, it is not trivial to predict how these parameters will change the PD outcome in practice. The PD algorithm is, in essence, trying to invert a complex and partially ambiguous imaging process. When estimating more WF coefficients, the reconstruction should, in principle, be more accurate, but it will work only up to a certain limit depending on the number and quality of the used recorded images. However, the additional images will also increase the computational demands, and when only moderate reconstruction quality is desired, two images with classical PD could be sufficient.

Next, we will illustrate in a few examples how the PD parameters and reconstruction error are coupled.

The PD algorithm is run with different numbers of estimated modal coefficients $\left(M+M^{\prime}\right)$; values of 26 , 36,66 , and 126 are used. We test the impact of additional WF information with $M^{\prime}=0$ and $M^{\prime}=20$, and we assume that there is no error when the WF coefficients are estimated from the WFS signal. In addition, two kinds of WFs are used: normal atmospheric turbulence with $r_{0}=0.08 \mathrm{~m}$ at $0.5 \mu \mathrm{m}$ and 
an AO-corrected WF. The AO correction is modeled by removing $40 \%$ of the WF rms contained in the 14 lowest KL modes.

We evaluate the PD performance by plotting the required number of FFTs versus image error. The image error is obtained by comparing the reconstruction and the original nonperturbed object as shown by Eq. (17). The results are shown in Fig. 3 .

From the results, we highlight the following points:

- When the classical 2-image PD is used without additional WF information, it is not necessary to estimate more than 36 modal coefficients to achieve the best possible image reconstruction $\left(\epsilon_{r} \approx 0.075\right)$ while seeing-limited error is $\epsilon_{s} \approx 0.11$.

- With the 4-image PD, and also when additional $\mathrm{WF}$ information is used, the image quality can be sig-
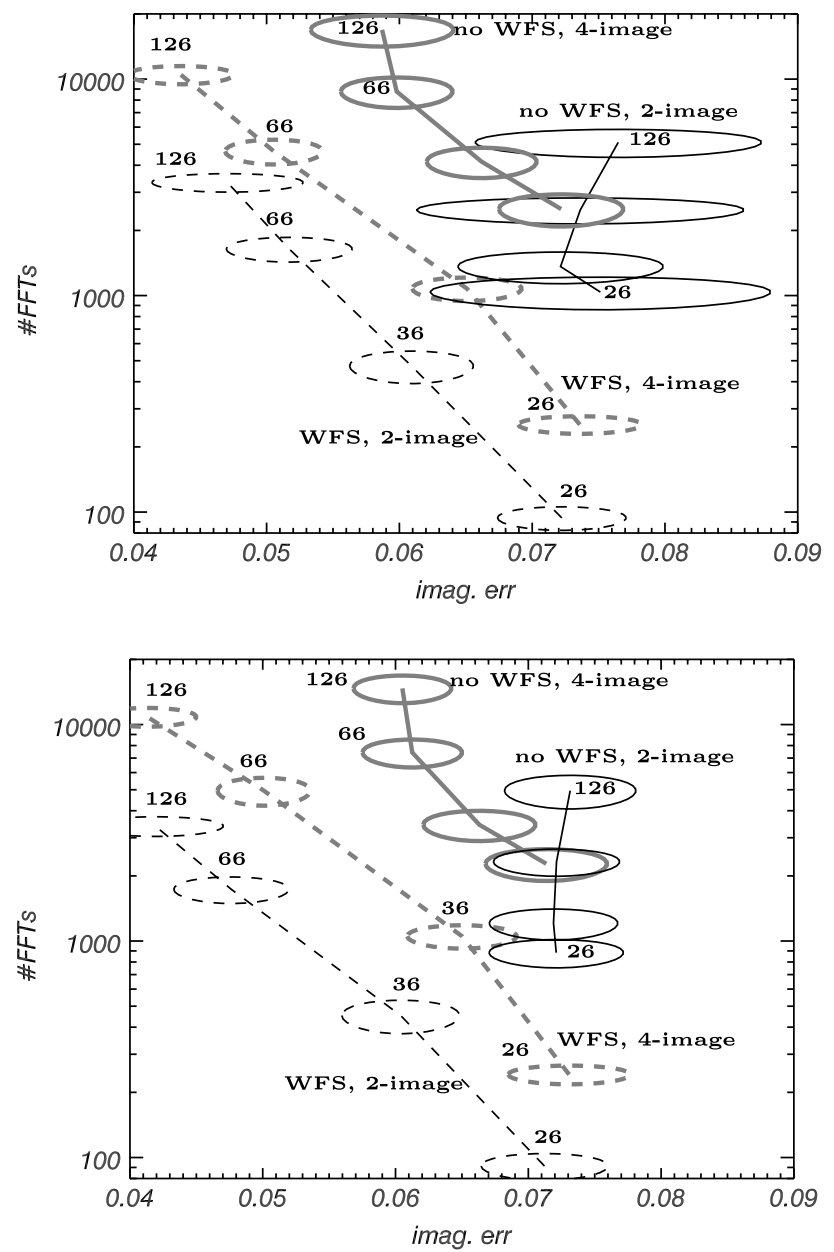

Fig. 3. The number of required FFTs as a function of reconstruction error. Solid lines show the pure PD; dashed lines are made with additional WF information. Thin lines are made with 2-image PD, thick lines with 4-image PD. The lines connect points with 26 126 estimated modal coefficients with the other simulation parameters fixed. The circles around the points show the rms computed over 32 random WF realizations and nine different subimages of the object. Upper plot: Kolmogorov-style turbulence $\left(r_{0}=0.08 \mathrm{~m}\right.$ at $0.5 \mu \mathrm{m}$ ). Seeing-limited image error $\epsilon_{s} \approx 0.11$. Lower plot: WFs typical for an AO system. AO-corrected image error $\epsilon_{s} \approx 0.09$. nificantly improved by increasing the number of estimated coefficients. The optimal quality is $\epsilon_{r} \approx 0.06$ without the WF knowledge and $\epsilon_{r} \approx 0.04$ with the WF knowledge. However, the computational costs increase exponentially with the image quality.

- To achieve a similar reconstruction quality to what the WFS-aided 2-image PD can optimally achieve $\left(\epsilon_{r} \approx 0.05\right)$, the conventional $\mathrm{PD}$ would require more than four images.

- The AO correction is able to improve the PD performance, in terms of rms image error, only by a negligible amount compared to the results obtained with additional WF information.

- Both 4-image PD and additional WF information, and also the AO correction, significantly improve the reliability of the image reconstruction: the rms of the achieved image quality is reduced by a factor of 2-3 compared to the conventional 2-image PD. Besides eliminating the worst cases, these techniques also remove the cases where the image error is the lowest.

- The additional WF information is extremely useful in reducing the computational demand of PD. Here, the computational load is reduced by a factor of $\sim 20$. It is seen that the additional WF knowledge enables a reliable use of the 2-image PD, which is the major reason for the lower computational need. However, the other reasons are also significant: less WF coefficients have to be estimated by the laborious $\mathrm{PD}$ algorithm, and the PD algorithm converges faster, when full knowledge of the low-order modal components in the WF is assumed.

In addition, we also tested different extents of $\mathrm{AO}$ correction by removing $10-100 \%$ of the WF rms contained by the lowest $14 \mathrm{KL}$ modes. It was found that the results were always very similar to what is shown in the lower plot in Fig. 3. The reliability of the classical 2-image PD was greatly improved, and the ultimate image reconstruction quality remained the same. Thus, the PD will work better with AO-corrected WFs, even when no lower spatial frequencies are present. Besides, this beneficial effect can be obtained with a very modest correction-it was enough to remove only $20 \%$ of the rms that was contained by low KL modes.

Based on these results, the best way to design a system using jointly $\mathrm{AO}$ and $\mathrm{PD}$ seems straightforward: correct as much turbulence as possible with the DM, and try to obtain as much WF information as possible with a WFS. If reliable WFS information is available, the quality of the DM correction is less important.

However, this analysis neglects two crucial issues: WF estimation errors and WF evolution between the instants when different diversities are applied. The next section will briefly cover these.

\section{Performance on realistic $\mathrm{AO}$ data}

The previous section has shown that the most advantageous scheme to jointly use $\mathrm{PD}$ with $\mathrm{AO}$ is applying PD directly for images that have been corrected by 
AO as much as possible. Unfortunately, with this strategy, it can be difficult to use additional WF information to aid the PD.

When working in a traditional, well-optimized, closed-loop operation, the single WFS measurements are very error-prone-the DM has already corrected most of the detectable aberrations, and a measurement of a residual phase thus represents the actual WF only with a limited accuracy.

However, it is also possible to run an AO system such that the DM introduces only diversities, but does not correct the turbulence-the upper plot in Fig. 3 illustrates an idealized case of this. In this way, the PD must deal with low-quality images, but relatively more accurate WF estimates are available, and they can potentially be used to reduce the computational demands of the PD task.

Next, we will illustrate through simulations how a realistic AO system would affect the $\mathrm{PD}$ performance. We simulate the AO with the CAOS software [30] and use the simulation data as the input for the PD algorithms. Two cases will be con- sidered, both with and without WFS assistance in the PD:

- PD based on pure atmospheric residuals. DM used only to create diversities. The WFs reconstructed using WFS information have WF rms errors of $27 \pm 7 \%$.

- Closed-loop AO. A typical AO loop is fully optimized. The estimated residual WFs have relative WF rms errors of $73 \pm 12 \%$, and the WF error compared to uncompensated WFs is $19 \pm 5 \%$.

In the latter case, the WFs are estimated with very poor relative accuracy, but on the other hand, the aberrations are much smaller.

The AO-corrected images have Strehl ratios of $0.26 \pm 0.05$ at $550 \mathrm{~nm}$, while the ratios in seeing are $0.05 \pm 0.01$. The WF error between consecutive frames, caused by the time-lag of $2.5 \mathrm{~ms}$, is typically $\sim 0.45 \mathrm{rad} \mathrm{rms}$ in the simulated seeing conditions.

In a low-order $\mathrm{AO}$ system like this, most of the WFS errors come from sources difficult to precisely characterize (aliasing, saturation) and from

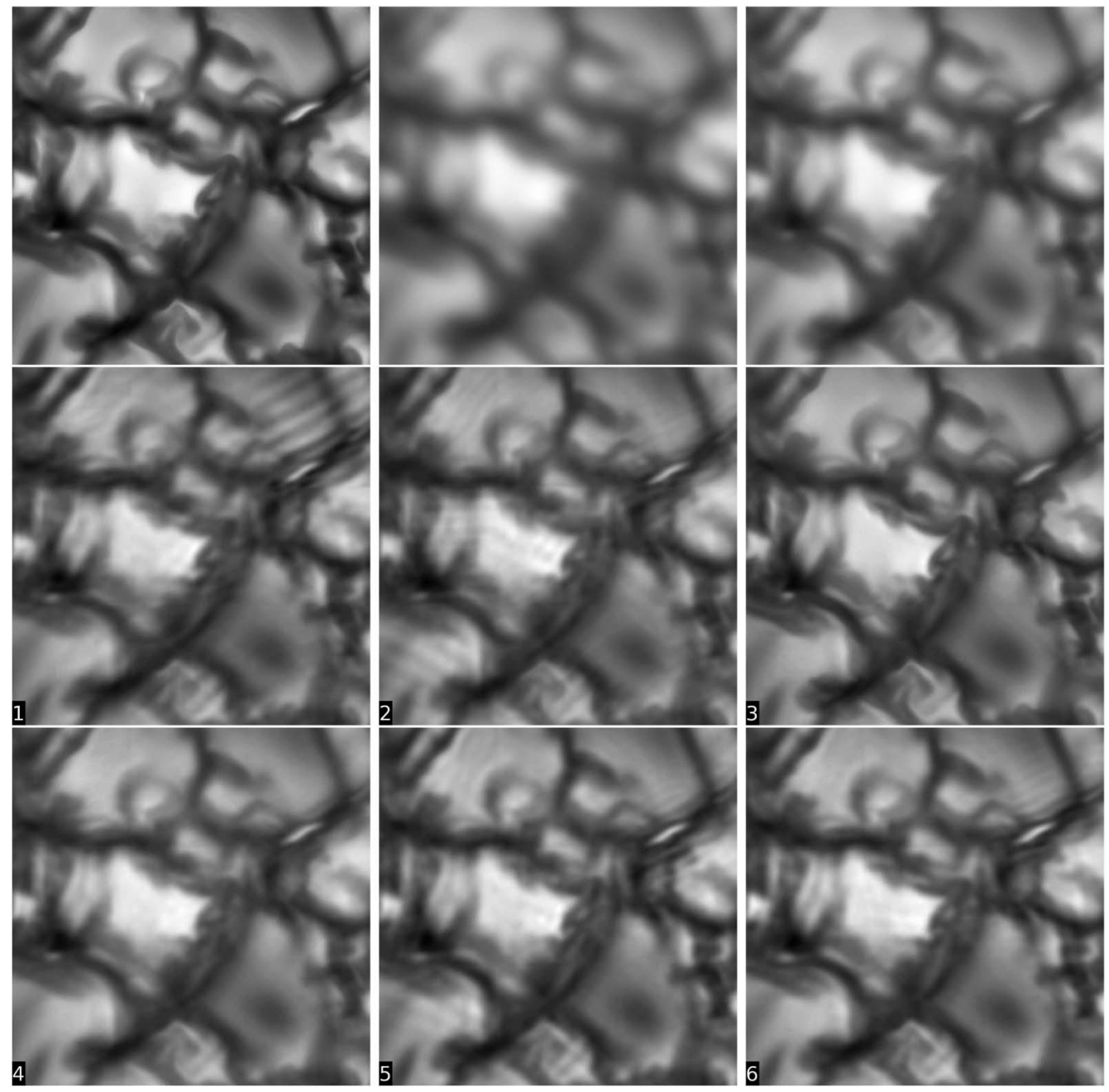

Fig. 4. Illustration of performance. All images have a dimension of $384 \times 384$ pixels, showing the region PD has reconstructed. Upper row: original object (having no spatial frequencies above the system diffraction limit), seeing-limited image, and AOcorrected image. Images 1-6 show examples of PD performance. See text for details. 
modal components the WFS misdetects. We have simulated the WF reconstruction with the standard CAOS software using point-sources, but we believe this also represents, with sufficient accuracy, the typical errors in our scenarios. A realistic system observing extended sources should implement the WFS as a correlation tracking, but we have postponed a more detailed analysis for a later stage.

We demonstrate the performance of the 2-image and 4-image PDs similar to how we did in the previous section. In addition, we study three modifications to the PD algorithm: no a priori information applied, WF coefficients initialized to values obtained from previous time-step, and also when the WF coefficients are estimated independently in two WFs $\left(K=2, \phi_{1}=\phi_{2}, \phi_{3}=\phi_{4}\right)$. In the independent estimation, we have two times more unknown WF coefficients, but the data collection is done identically to the 4-image $\mathrm{PD}$.

We study the PD performance in terms of a few measures: improvement compared to seeing $\left(\epsilon_{r} / \epsilon_{s}\right)$, WF reconstruction error, fail rate (definition of failure defined in Table 3), number of required FFTs, and PD iterations. The performance measures and their ranges, defined as rms over 256 consecutive time steps and the $3 \times 3$ reconstructed subimages, are shown in Table $\underline{3}$.
It is seen that PD is working well also in the presence of significant time-lag (delay between the sequential images). With 2-image $\mathrm{PD}$, the relative image error, $0.50-0.63$, falls exactly into the range expected from the ideal assumptions in Subsection 3.B-the performance loss is negligible.

The fail rates, however, describe the situation slightly better. If $5-10 \%$ of the frames will be incorrectly reconstructed, the usefulness of the system becomes questionable. Conventional 2-image PD has serious issues even when WFS information is available. This happens both with and without the $a$ priori information of the WFs.

When the 4-image PD is used, we again reach the performance exactly as expected based on the ideal simulations. We see that the error drops down to 0.42 , and $0 \%$ fail rate can be achieved. This happens even though the WF estimation errors caused by phase-lag increase when more images are used.

It is also seen that the PD is working extremely well with the $A O$ residuals. In that regime, the WFS-aided PD also produces excellent results: the image error is reduced well below what is achieved by pure AO alone-although the low-order modal coefficients, estimated from WFS information, have a huge estimation error of $73 \pm 12 \%$ in WF rms. This happens because the PD error metric does not optimize the WF, but the image quality.

Table 3. PD Performance on AO Sequences

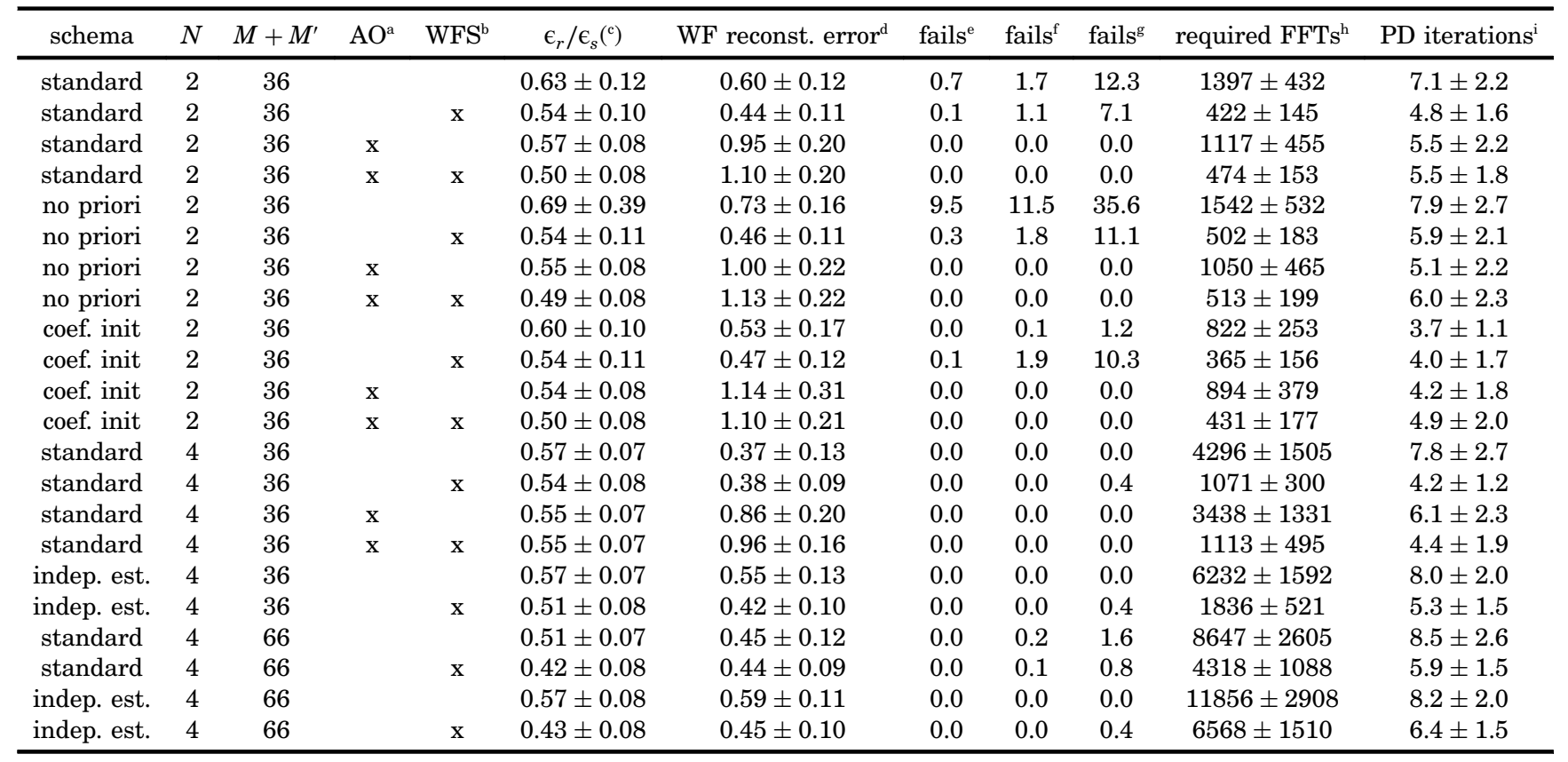

${ }^{\text {aPD }}$ applied to closed-loop WF residuals

${ }^{b}$ WFS aid used in PD

${ }^{c} \mathrm{PD}$ reconstruction error $\left(\epsilon_{r}\right)$ compared to original seeing-limited error $\left(\epsilon_{s}\right)$

${ }^{\mathrm{d}}$ Relative error in reconstructed WF, $\hat{\phi}_{r} / \phi$; only $M+M^{\prime}$ modal components considered

${ }^{\mathrm{e}}$ Fail rate, percentage of cases when $\epsilon_{r}>\epsilon_{s}$

${ }^{\mathrm{f}}$ Fail rate, percentage of cases when $\epsilon_{r}>\left(\tilde{\epsilon}_{s}+\tilde{\epsilon}_{r}\right) / 2$, where $\tilde{r}$ denotes a momentary temporal average

${ }^{g}$ Fail rate, percentage of cases when the condition ${ }^{\mathrm{f}}$ is fulfilled at least in one subimage at a given time-step

${ }^{\mathrm{h}}$ Number of FFTs for each considered subimage with a size of $256 \times 256$

${ }^{i}$ Number of PD iterations for each considered subimage 
A similar phenomenon has been reported by [4,31]: $\mathrm{PD}$ can find better reconstructions compared to what is obtained when the deconvolution is done using a perfect modal WF representation being truncated to as many coefficients. Here, we observe a variation of the same effect. The PD-estimated high-order coefficients (KL modes higher than 20) can also compensate the errors of the lowest modes-we verified this by checking that the perfect truncated WFs gave worse performance. This is also reflected in the large errors of estimated WFs: they can be estimated at best with an accuracy of $\sim 40 \%$, although PD can be successful even with errors greater than $100 \%$.

Thus, it can be concluded that, in the regime we are studying here, the loss of information in the noninvertible imaging process is the dominant error source in PD by a huge margin. A typical phaselag will not significantly disturb the PD outcome, and the PD does ssignificantlybenefit from the WFS aid, although the used phase-estimates can have very significant WFS errors.

Therefore, it is not surprising that when the WFs at different time steps are estimated independently, the PD results are not improved. However, we can see that the reconstruction is slightly more robust with a large number of estimated modes (66): the strictest fail rate is reduced to zero from $0.8-1.6 \%$.

Regarding the computational demand, it is seen that again, the WFS information and closed-loop AO can simplify the task significantly. If the goal is $50 \%$ reduction in the image error, the computational costs are reduced by a factor of almost 20 .

The most significant factor in the reduction is the lower amount of modal coefficients that need to be estimated by PD. The number of PD iterations is also reduced, from $\sim 8$ to $4-5$, and thus it can reduce the number of required FFTs by a factor of 2 . However, the possibility to initialize the modal coefficients to values obtained from a previous frame seems not to be a huge factor in the case we are studying. When WFS-aided PD is used with closed-loop AO, the initialization helps to reduce the computational costs only by $10-20 \%$. If no WFS or AO is used, the initialization is more helpful, and it can reduce the needed FFTs by $\sim 40 \%$.

Finally, we illustrate the image quality after PD through a few practical examples. The upper row in Fig. 4 shows the original object and how it is observe $\bar{d}$ in seeing-limited $\left(\epsilon_{s}=0.118\right)$ and $\mathrm{AO}$ corrected $\left(\epsilon_{s}=0.079\right)$ conditions. The parameters are the same as in Table 2. The next two rows illustrate how well the image can be reconstructed in six selected cases:

1. traditional 2-image $\mathrm{PD}, M=36\left(\epsilon_{r}=0.070\right.$, $\left.\epsilon_{r} / \epsilon_{s}=0.59\right)$,

2. WFS aid used with 2-image PD, $M=16, M^{\prime}=$ $20\left(\epsilon_{r}=0.070, \epsilon_{r} / \epsilon_{s}=0.59\right)$,

3. AO closed-loop residual and WFS aid used with 2-image $\mathrm{PD}, M=16, M^{\prime}=20 \quad\left(\epsilon_{r}=0.052\right.$, $\left.\epsilon_{r} / \epsilon_{s}=0.44\right)$,
4. the 4-image $\mathrm{PD}, \quad M=66 \quad\left(\epsilon_{r}=0.061\right.$, $\left.\epsilon_{r} / \epsilon_{s}=0.51\right)$,

5. and WFS aid used with 4-image $\mathrm{PD}, M=46$, $M^{\prime}=20\left(\epsilon_{r}=0.047, \epsilon_{r} / \epsilon_{s}=0.39\right)$.

6. Two WFs are estimated independently with the 4-image $\mathrm{PD}, \quad M=46, \quad M^{\prime}=20 \quad\left(\epsilon_{r}=0.050\right.$, $\left.\epsilon_{r} / \epsilon_{s}=0.42\right)$.

AO, when applied alone, significantly improves the image resolution, but based on visual inspection, it is not reaching the same quality as the PD. Even traditional 2-image PD has more details available in the image (image 1). However, the traditional PD has serious sinusoidal artifacts, in particular at the top and right of the example image. Similar phenomena have been observed, for instance, by [4].

When WFS information is included (image 2), the number of artifacts is significantly reduced, although on average the relative image error (c) does not improve for the particular frame shown in Fig. $\underline{4}$. Also, visual inspection does not reveal any significant improvement in the image resolution.

When the 2-image PD is applied to AO-corrected closed-loop images (image 3), clearly the best result is obtained. Visual inspection does not reveal any ignificant artifacts, and the image resolution is comparable to the original object.

If no AO or WFS aid is available, a comparable image error, in terms of rms, can be reached by the 4image PD and 66 estimated modes. One example is illustrated by image 4 . It has no artifacts, but the visual inspection reveals slightly more smearing of the small details compared to image 3 . In addition, it requires 15-20 times more computation to reconstruct.

Image 5, obtained with WFS-aided 4-image PD, has the lowest relative image error $\left(\epsilon_{r}=0.047\right)$. However, it is seen that small artifacts emerge again in the top-right corner of the image. This probably happens because of the incorrect WFS estimates, as no artifacts were seen in image 4 . Similar artifacts are also present in image 6 , which shows the result when the WFs were estimated independently.

\section{Conclusions}

This work demonstrates, through numerical simulations, the potential that $\mathrm{PD}$ and $\mathrm{AO}$ can have, when used together sequentially. We have seen that such potential indeed exists in the cases we studied: both $\mathrm{AO}$ and $\mathrm{PD}$ applied together will produce better results than one of them alone.

In addition, we identified a few areas where the benefits of the joint optimization should be the easiest to realize.

First, it was seen that the PD also works extremely well with AO-corrected phase residuals. The AO correction helps to make the reconstruction robust, although not much can be gained in the reconstruction error in terms of rms. In addition, only a small amount of correction is needed: it was necessary to correct only $20 \%$ of the low-order modes to obtain a near-optimal performance. Correcting more still improves results, but only slightly. 
Second, the additional WFS information was observed to be extremely useful in both making the image reconstruction more robust and reducing the computational demands. In the case we studied, it was possible to reduce the computational costs by a factor of $\sim 20$ without loss in performance, minor artifacts excluded. This will be extremely important when using $\mathrm{PD}$ in real-time, or close to real-time.

Third, the magnitude of the applied diversity, created by the DM, can be adjusted based on the seeing conditions. The simulations indicate that within a seeing range of $0.65-2$ arcsec, where the WF distortions are on the order of 3-7 rad rms, a good reconstruction can be obtained with all diversity shapes having an rms of 4-10 rad. However, this requires the computationally demanding 4-image PD. With the computationally less demanding 2 -image $\mathrm{PD}$, or when more modal components are estimated, more care should be taken to optimize the applied diversity magnitude-otherwise the image error can be 5-20\% higher.

Finally, it was shown that the error sources typical for a realistic AO system appear negligible. All the important performance gains were obtained in spite of the large WFS errors and the time-lag between subsequent images.

Our future work will concentrate on demonstrating the results in a laboratory setup. Several possible issues can still prove challenging in implementing a successful AO-PD system. For instance, the DM accuracy when producing the diversity is crucial for $\mathrm{PD}$ algorithms to work. Based on the results, however, we believe that minor errors in the WF accuracy will not cause dramatic problems.

If the imaging is recorded with a wide wavelength bandwidth, some modifications of the models might be necessary to take into account the additional blurring of the speckles in the PSF. In addition, more work is needed to fine-tune the algorithms to optimize the performance. Reliable methods are needed to select the PD parameters such as the number of coefficients and the number of independent WFs. Besides, more detailed analysis on the WFS errors should be included to optimize the number of WFSestimated coefficients.

Regarding the final system performance, however, the most critical issue might be the anisoplanatic errors. The WFS measures the distortions only in one direction, and thus the WF estimates are valid only in a small subregion of the corrected field. Similarly, the DM corrections would not improve, but rather degrade, the image quality outside of the direction where the AO is locked. This issue will be addressed in our future work, including experiments at relevant sites.

We thank A. Vögler and N. Vitas for providing the synthetic solar images.

\section{References}

1. F. Roddier, Adaptive Optics in Astronomy (Cambridge University, 1999).
2. R. A. Gonsalves, "Phase retrieval and diversity in adaptive optics," Opt. Eng. 21, 829-832 (1982).

3. R. G. Paxman, T. J. Schulz, and J. R. Fienup, "Joint estimation of object and aberrations by using phase diversity," J. Opt. Soc. Am. A 9, 1072-1085 (1992).

4. M. G. Löfdahl and G. B. Scharmer, "Wavefront sensing and image restoration from focused and defocused solar images," Astron. Astrophys. Suppl. Ser. 107, 243-264 (1994).

5. R. G. Paxman, J. H. Seldin, M. G. Löfdahl, G. B. Scharmer, and C. U. Keller, "Evaluation of phase-diversity techniques for solar-image restoration," Astrophys. J. 466, 1087-1099 (1996).

6. M. G. Löfdahl, "Multi-frame blind deconvolution with linear equality constraints," Proc. SPIE 4792, 146-155 (2002).

7. M. van Noort, L. R. van der Voort, and M. G. Löfdahl, "Solar image restoration by use of multi-frame blind de-convolution with multiple objects and phase diversity," Sol. Phys. 228, 191-215 (2005).

8. J. Sauvage, T. Fusco, G. Rousset, and C. Petit, "Calibration and precompensation of noncommon path aberrations for extreme adaptive optics," J. Opt. Soc. Am. A 24, 2334-2346 (2007).

9. S. M. Jefferies, M. Lloyd-Hart, E. K. Hege, and J. Georges, "Sensing wave-front amplitude and phase with phase diversity," Appl. Opt. 41, 2095-2102 (2002).

10. T. Berkefeld, "Solar adaptive optics," in Modern Solar Facilities-Advanced Solar Science, F. Kneer, K. G. Puschmann, and A. D. Wittmann, eds. (Universitätsverlag Göttingen, 2007), pp. 107-113.

11. F. J. Rigaut, B. L. Ellerbroek, and R. Flicker, "Principles, limitations, and performance of multiconjugate adaptive optics," Proc. SPIE 4007, 1022-1031 (2000).

12. J.-P. Véran, F. Rigaut, H. Maître, and D. Rouan, "Estimation of the adaptive optics long-exposure point-spread function using control loop data," J. Opt. Soc. Am. A 14, 3057-3069 (1997).

13. C. U. Keller, T. R. Rimmele, R. G. Paxman, J. H. Seldin, D. Carrara, and K. Gleichman, "Evolution of small-scale magnetic fields from combined adaptive optics and phase-diverse speckle imaging," in AAS / Solar Physics Division Meeting \#31, vol. 32 of Bulletin of the American Astronomical Society (American Astronomical Society, 2000), p. 833.

14. M. G. Löfdahl and G. B. Scharmer, "Phase diverse speckle inversion applied to data from the Swedish 1-meter solar telescope," Proc. SPIE 4853, 567-575 (2003).

15. K. G. Puschmann and M. Sailer, "Speckle reconstruction of photometric data observed with adaptive optics," Astron. Astrophys. 454, 1011-1019 (2006).

16. J. H. Seldin, R. G. Paxman, D. A. Carrara, C. U. Keller, and T. R. Rimmele, "Deconvolution of narrowband solar images using aberrations estimated from phase-diverse imagery," Proc. SPIE 3815, 155-163 (1999).

17. A. Blanc, T. Fusco, M. Hartung, L. M. Mugnier, and G. Rousset, "Calibration of NAOS and CONICA static aberrations. Application of the phase diversity technique," Astron. Astrophys. 399, 373-383 (2003).

18. R. A. Gonsalves, "Adaptive optics by sequential diversity imaging," in Beyond Conventional Optics, E. Vernet, R. Ragazzoni, S. Esposito, and N. Hubin, eds. (European Southern Observatory, 2002), Vol. 58, pp. 121-124.

19. R. A. Gonsalves, "Sequential diversity imaging: phase diversity with AO changes as the diversities," in Frontiers in Optics, Technical Digest (CD) (Optical Society of America, 2010) p. FWV1.

20. A. W. van Eekeren, K. Schutte, J. Dijk, and P. B. Schwering, "Time-varying phase diversity turbulence compensation," Proc. SPIE 8012, 80120D (2011).

21. F. Roddier, "Theoretical aspects," in Adaptive Optics in Astronomy, F. Roddier, ed. (Cambridge University, 1999), pp. 25-56.

22. R. J. Noll, "Zernike polynomials and atmospheric turbulence," J. Opt. Soc. Am. A 66, 207-211 (1976).

23. E. Peli, "Contrast in complex images," J. Opt. Soc. Am. A 7, 2032-2040 (1990).

24. E. V. Khomenko, S. Shelyag, S. K. Solanki, and A. Vögler, "Stokes diagnostics of simulations of magnetoconvection of 
mixed-polarity quiet-Sun regions," Astron. Astrophys. 442, 1059-1078 (2005).

25. V. Korkiakoski, C. Vérinaud, and M. Le Louarn, "Improving the performance of a pyramid wavefront sensor with modal sensitivity compensation," Appl. Opt. 47, 79-87 (2008).

26. M. W. Smith, "Use of adaptive optics to implement nonquadratic phase diversity imaging," Proc. SPIE 5524, 66-77 (2004).

27. D. J. Lee, M. C. Roggemann, and B. M. Welsh, "Cramér-Rao analysis of phase-diverse wave-front sensing," J. Opt. Soc. Am. A 16, 1005-1015 (1999).

28. L. Meynadier, V. Michau, M.-T. Velluet, J.-M. Conan, L. M. Mugnier, and G. Rousset, "Noise propagation in wave-front sensing with phase diversity," Appl. Opt. 38, 4967-4979 (1999).
29. J. J. Dolne, R. J. Tansey, K. A. Black, J. H. Deville, P. R. Cunningham, K. C. Widen, and P. S. Idell, "Practical issues in wave-front sensing by use of phase diversity," Appl. Opt. 42, 5284-5289 (2003).

30. M. Carbillet, C. Vérinaud, B. Femenía, A. Riccardi, and L. Fini, "Modelling astronomical adaptive optics-I. The software package CAOS," Mon. Not. R. Astron. Soc. 356 , 1263-1275 (2005).

31. G. B. Scharmer, M. G. Löfdahl, T. I. M. van Werkhoven, and J. de la Cruz Rodríguez, "High-order aberration compensation with multi-frame blind deconvolution and phase diversity image restoration techniques," Astron. Astrophys. 521, A68 (2010). 